\title{
Study on Transformation and Upgrading of Urban Agglomeration in Guanzhong Plain
}

\author{
Songbai Zhang \\ Economy and Management, Longdong University, Qingyang, Ganshu, 745000
}

Keywords: technical innovation; industrial structure; urban agglomeration

\begin{abstract}
The urban agglomeration in Guanzhong Plain has some systemic problems, such as small scale of core city, lack of big city, small number of medium cities, and convergent industrial structure, unable to play a good role in agglomeration and radiation. Only by choosing a scientific and effective path can the transformation and upgrading of urban agglomeration be completed, such as optimizing the industrial structure in the cluster, strengthening the role of the core city, filling the blank of the big city with the technological innovation and the advantage of modern network information, etc.
\end{abstract}

\section{The role and significance of urban agglomeration transformation and upgrading in Guanzhong Plain}

\subsection{It is the objective requirement of the theory and practice of unbalanced development}

The theory of unbalanced development holds that balance is conditional, relative and temporary, and the unbalanced economic development between regions is objective. Absolutely, the economic development of the region must be dynamic, and there must be an imbalance of development and the inevitability of the unbalanced development of the region ${ }^{[1]}$. In the process of regional economic development, the local economic development will be more rapid than the location conditions, the gap between the developed and the underdeveloped areas will be further widened. However, in this process, the balance force will take place, and the development of the developed regions will lead to the development of the underdeveloped areas. In order to get rid of the difficult situation, the region will give full play to its comparative advantage and the developed region, which will form a kind of force so that the gap will not be further widened. Therefore, it is the objective requirement of the unbalanced development law from the unbalanced development to the balanced development ${ }^{[2]}$. As far as the northwest region is concerned, urban agglomeration in Guanzhong has a long history, rich accumulation, concentrated city, good industrial foundation and profound cultural background, and has the basis for the whole development of the whole northwest region.

1.2 It is the fruit of the great development of the western region and the new starting point for the revitalization of the western region

Since the founding of New China, every tilt and focus of the state policy will promote the rapid development of the regional economy. The "three-line construction" in the 1960s changed to a certain extent the economic distribution of the original industries concentrated in non-raw material producing areas, and promoted the full exploitation and utilization of natural resources in the central and western regions. A strong production base and a number of sophisticated experimental bases have been established in the west, promoting the coordinated economic development of the east and the west. The development of the western region began in 2000. After nearly ten years of development, the economic indexes of the western region were greatly improved. From 2000 to 2008, the gross domestic product of the western region increased from 1.6655 trillion Yuan to 5.8257 trillion Yuan, with an average annual growth rate of 11.7 Yuan, which accounted for 17.1 percent of the total proportion of the regions increased to 17.8 percent. The per capita regional gross domestic product increased from 4624 Yuan to 16000 Yuan. ${ }^{[3]}$ the 13th Five-Year Plan for the Great 
Development of the Western region has even formulated a new goal: to build a number of representative innovative cities and regions, and to raise the level from $59.5 \%$ to $63.7 \%$ of the total average level of each region. ${ }^{[3]}$ The 13th Five-Year Plan for the Great Development of the Western region has even formulated a new goal: to build a number of representative innovative cities and regions. The formation of an advantageous industrial system with the characteristics of the western region, and thus drive the national economic development. ${ }^{[4]}$ "Guanzhong Plain Urban agglomeration Construction Plan" is the new era and the new starting point for the development of the western region under the new conditions.

\subsection{It is a strategic support point for the construction of the "Silk Road"}

From a horizontal point of view, the Silk Road economic belt presents the three major regions in the east, the central and western regions, with a gradient distribution of development levels, and the east is a rapidly developing Asia-Pacific economic circle. The west is a relatively developed European economic circle, and the central and south Asian regions form an economic depression zone. There are significant differences between the East, West and West regions. The East and Southeast Asian countries of the eastern plate have relatively complete industrial systems and capital. Rich in technology and labor resources, it is the export place of industrial products, but most of the industries are at the mid-level of the global industry. European countries are advanced in science and technology, industrial development, and occupy the high end of $\mathrm{R} \& \mathrm{D}$, manufacturing and intellectual property rights. Holding the high value-added income chain under globalization, but the main resources are scarce, so we need to expand the space for development abroad; the Middle East, North Africa and Central Asia mainly rely on oil exports for economic development in the Middle East, North Africa and Central Asia, while the economic level of West Asia and Central Asia is relatively backward. The industrial system is unitary. Except for some countries with a certain industrial base, they are generally in the traditional manufacturing industry and the low-end resource export industry, but are rich in resources such as energy, minerals, etc. This is also a key area of development in cooperation with other countries along the Silk Road Economic Belt. Economy is in a period of rapid development in China, and there is an exuberant demand for high and new technology, management methods and resources in the central plate of the western plate. Its excess production capacity and capital is the improvement of infrastructure in countries along the Silk Road, and has high quality resources for revitalizing our economy. China is the initiator and practitioner of the Belt and Road initiative and the western part of China is the strategic support point connecting the eastern plate and the central and western plate. From the domestic point of view, the natural conditions in the east of Guanzhong are better, the economic elements flowing actively, the foundation of economic development is good, and the speed is fast. The main space of economic growth in China is distributed in this area. The west of Guanzhong is the western region with abundant resources and vast area. The eastern industries should be transferred to the west, and Guanzhong region is an important region for undertaking and transit. The quality and level of Guanzhong urban agglomeration is directly related to the industrial transfer from east to west of China.

\section{The main problems of urban agglomeration in Guanzhong Plain}

\subsection{The large cities are absent, the middle cities are few, and the urban agglomeration and radiation functions are not well brought into play}

Its hierarchical structure should have five levels: mega cities, medium cities, and small towns. The overall scale of Guanzhong urban agglomeration is small. Compared with Xian as a mega-city, Baoji is higher than other medium-sized cities in comprehensive urban indicators, but it cannot be counted as a big city in the region. Xianyang, Tongchuan, Weinan, Shangluo, Yuncheng, Linfen, Tianshui, Pingliang, Qingyang are medium cities. The discontinuity of city grade affects the range of the agglomeration effect and diffusion effect of Xian as the central pole, which makes the function of Xian can not spread to the whole urban agglomeration system in turn through the urban 
network. Due to the great difference between their own economic strength and that of mega cities, it is difficult for medium cities to effectively absorb and digest Xian industries, products and technology, and at the same time, there is no way to directly provide Xian with a perfect resource base and auxiliary industries. In particular, Pingliang in Gansu Province, Yuncheng in Qingyang and Yuncheng in Shanxi Province, Linfen is far from the hinterland of Guanzhong, major transport facilities are under construction (such as Yinxi high-speed rail), and the obstacles of administrative ownership make it possible to absorb Xian products, and the ability to transfer technology is limited. Radiation effects on small cities and towns are difficult to play. The scale of the core cities is not large enough; the integration of Xian should speed up the pace of the total population of Xian in 2016, 8.2493 million people or 625.718 billion Yuan of gross domestic product (GDP), Xianyang total population of 4.9866 million people (GDP) 239.007 billion Yuan. The total population of Guangzhou in the Pearl River Delta was 1.954744 trillion Yuan (GDP) at the end of 2016, and the total population of Shanghai in the Yangtze River Delta was 2.817865 trillion Yuan at the end of 2016. The total population of Beijing around the Bohai Sea was 2.566913 trillion Yuan (GDP) at the end of 2016, and that of Tianjin (1.788539 trillion Yuan) was larger than that of Xian. The scale of the core city represents the status of the urban agglomeration. The total population of Chongqing in the Chengdu-Chongqing urban agglomeration was 1.774059 trillion Yuan at the end of 2016, and the total population of Chengdu at the end of 2016 was 1398.9 Yuan, 1.217023 trillion Yuan, which is much larger than that of Xian. The status of Guanzhong urban agglomeration in the west of China is lower than that of Chengdu-Chongqing urban agglomeration. "Guanzhong Plain Urban agglomeration Construction Plan". Xian, Xianyang main urban district and Xianxian New area constitute the Xian Metropolitan Circle, which makes Xian and Xianyang integrated development. Create a modern metropolitan area with international influence. But adjusting the administrative division, optimizing the function layout and strengthening the core function of the city still need to do a lot of hard and complicated work.

\subsection{The urban industrial structure converges and does not form the modern industrial system of division of labor and cooperation}

The industrial division of labor in Guanzhong urban agglomeration has a serious convergence phenomenon, and it is an extensive economic growth mode. According to the correlation coefficient between the economy of Guanzhong urban agglomeration and the development of resources, there is a positive correlation between the increase of economic system level and the increase of resource system level, and every unit added to the economic system requires an increase of 1.4 units in the resource system. From this we can see that Guanzhong economic growth mode is still high input, high consumption of extensive growth. The 5 cities of Guanzhong plain urban agglomeration, which belong to Shaanxi, are the pillar industries of machinery, electronics and textile. The industrial division of labor has not formed a grads level. The homogeneous competition between small and medium-sized cities is outstanding. In particular, the similarity coefficient of industrial structure between Baoji and other cities is up to 0.9, and the industrial isomorphism of is obvious. The industrial development and layout have not fully reflected the comparative advantage and resource endowment of each city, especially the vertical and horizontal division of labor in each city is not clear, and the industrial complementarities are more reflected in the difference of function and scale. There is no vertical industrial relationship between leading and supporting, which leads to the poor coordination degree of the functional structure within the urban agglomeration, which further weakens the economic relationship between cities and affects the overall development of the urban agglomeration.

\section{The route of transformation and upgrading of urban agglomeration in Guanzhong Plain}

\subsection{Optimize the industrial structure of urban agglomeration in Guanzhong}

The optimization of industrial structure is to change the structure by input of some kind of signal and energy, thus forming the process of the new industrial structure. According to the nature of the 
input signal and the type of adjustment, the coordination mechanism of the market and the coordination mechanism of the government can be chosen by the industrial coordination mechanism of the urban agglomeration in Guanzhong. The industrial structure of the market mechanism is to adjust the industrial structure of the economic subject under the guidance of the market signal and make the industrial restructuring conform to the endowment of the production factors in various regions. Due to the difference in the factor endowment in Guanzhong area, it determines the difference between the industrial structure of a city and the other urban industrial structures. For example, the high quality of the labor force in Xian is conducive to the development of the technology intensive type. Or knowledge intensive industries, forming a regional industrial structure dominated by technology or knowledge intensive industries; Tongchuan is rich in mineral resources, and is conducive to the development of resource based industries and the formation of a regional industrial structure dominated by resource based industries.

The government regulation mechanism is an active adjustment process of the economic system, that is, the government directly carries out the allocation of resources in the industry through administrative or economic means, so that the industrial structure is reasonable. Regardless of the market coordination mechanism or the government coordination mechanism, the structure layout of the industrial sector should be optimized from the overall goal of the economic development and to the economic subject through the vertical hierarchical level of the urban agglomeration.

\subsection{Further Strengthen the Central Role of Xian for the Core City}

Xian is the central city of Guanzhong urban agglomeration and the western region of China. It has the advantages of advanced science and education, strong comprehensive industrial ability and rich tourist resources. In the future, we should strive to develop into the original base of industry, product and technology in Guanzhong urban agglomeration. In particular, on the basis of science planning, it should include traffic and communication construction, network Construction, environmental governance and other content infrastructure construction as the starting point, strengthen urban function and improve the modernization level; take the domestic and international market demand as the guidance, highlight the advantages, promote the upgrading of the product structure, develop the small and medium enterprises, expand the economic scale, construct the special economy, and develop the high and new technology industry and the modern service industry. We should Focus on the development of high-tech industries and modern service industries, intensify the efforts to transform traditional industries with high-tech industries, optimize and upgrade the industrial structure, promote enterprises to truly become the main body of technological innovation, and form international financial centers and commodities. The main factor market absorbs the capital, technology, management experience and transfer industry of the eastern region and foreign countries, and radiates, penetrates and drives the large and medium cities in the urban agglomeration.

\subsection{Fill the gap of big cities with technological innovation and modern network information superiority}

From the overall structure of the Guanzhong urban agglomeration, there is a lack of large cities which can connect the mega cities and the middle cities, so filling the gap in the big cities is an important content of the coordinated development of the urban agglomeration in Guanzhong. However, the development of the city has its own regularity. Under the technical conditions, it is possible to fill the gap of the lack of large cities by technological innovation and modern network information superiority, and explore a development model of urban agglomeration with the characteristics of Guanzhong. The higher institutions and scientific research institutes have a relatively obvious advantage of research and development, and the high technology industry added value index ranks in the top of the country, and the western part of the country is in the forefront of the country, and the western part of the high technology industry is in the forefront of the country. The urban agglomeration in Guanzhong should make up for the short, strengthen the motivation of the study and innovation of the urban agglomeration, give full play to the advantages of the environment and organization, give birth to the innovation subject, make it a progressive force to 
promote the economic development of the urban agglomeration, and make full use of the modern information technology to promote the interrelation of these scientific and technological resources. To strengthen the construction of the chain of industry, science and research, strengthen the enterprise grasp of the market and technical direction, promote the cooperation and innovation of the related enterprises, speed up the speed of innovation, make up for the shortcomings of the lack of large cities, and explore a new and no precedent for the development model of the Guanzhong urban agglomeration.

\subsection{Increase the number of medium-sized cities and vigorously develop characteristic towns}

There are 10 middle cities in Guanzhong urban agglomeration: Baoji, Xianyang, Tongchuan, Weinan, Shangluo, Yuncheng, Linfen, Tianshui, Pingliang and Qingyang. The planning area is large and the number of cities is small, which requires the improvement of the level of middle cities. At the same time, we should continue to supplement the number of medium-sized cities, and increase the population of medium sized cities with population around 300 thousand as the main contents of the construction of Guanzhong urban agglomeration. Whether small cities and towns can flourish is whether it has its own characteristics and whether it can adapt to the needs of local social and economic development. The small cities and towns in the Guanzhong urban agglomeration are small, the level of development lags behind, and many rural residents live in the difficult mountainous areas, and the education and medical treatment are backward, and the natural and semi natural economic characteristics are found. It is obvious that we should rationally plan and guide these small towns, develop them into tourist towns with distinctive characteristics, specialties, small towns, cultural towns and so on.

\section{References}

[1] Lin Yinglu. Theory of unbalanced Development and Economic Development Strategy of Western China. [J]. The influence of Science on Society. 1996 (4):34-37

[2] Gao Dawei. Review on the Theory of unbalanced Development of Regional economy [J]. The shopping mall is modernized. Academic Edition. 2005 (7):143-144

[3] Achievements in the Great Western Development. [EB/OL].

https: / zhidao.baidu.comdu.comr.question.383623767.html.2018.3.29

[4] The NDRC issued the "13th Five-Year Plan for the Great Western Development" [full text] [EB/OL]. http://www.h2o-china.com/news/252959.html.2010.1.25. 15:20

[5] Evolution characteristics and Optimization Strategy of Urban system in Guanzhong Plain Urban agglomeration [J]. Macroeconomic management. 2018 (1):72-76 